\title{
Investigation of elastic wave transmission in a metaconcrete slab
}

\author{
Stephanie J. Mitchell ${ }^{a}$, Anna Pandolfi ${ }^{\mathrm{b}, *}$, Michael Ortiz ${ }^{\mathrm{a}}$ \\ ${ }^{a}$ EAS Division, California Institute of Technology, Pasadena, CA 91125, USA \\ ${ }^{\mathrm{b}}$ DICA, Politecnico di Milano, 20133 Milano, Italy
}

\section{A R T I C L E I N F O}

\section{Article history:}

Received 9 March 2015

Received in revised form 31 July 2015

Available online 2 September 2015

\section{Keywords:}

Metaconcrete

Transmission coefficient

Resonant aggregate

Negative mass

Metamaterials

\begin{abstract}
A B S T R A C T
A new type of modified concrete, termed metaconcrete, has been shown to exhibit trapping of wave energy and a reduction in mortar stress when subjected to dynamic loading. Metaconcrete replaces the standard stone and gravel aggregates of regular concrete with spherical inclusions consisting of a heavy core coated with a compliant outer layer. These new layered aggregates resonate at designed frequencies by allowing for relative motion between the heavy core and the mortar matrix, which causes the aggregate to absorb energy and therefore reduce stress within the mortar phase of the composite material. The transmission of wave energy through a metaconcrete slab can be used to visualize the effect of resonant behavior within the metaconcrete aggregates. To quantify this behavior we compute a transmission coefficient, which is a method of measuring the absorption of wave energy as an applied forcing of known frequency travels through the material. The transmission coefficient is plotted against forcing frequency for four different aggregate material and geometry configurations. A reduction in transmission ratio is observed at or near the computed natural modes of the aggregate, indicating the activation of resonance within the inclusions. This behavior is consistent with observations from studies on sonic metamaterials containing resonant inclusions with a similar layered structure. The frequency location and width of the dip in transmission coefficient will aid in the design of metaconcrete aggregates for specific forcing applications.
\end{abstract}

(c) 2015 Elsevier Ltd. All rights reserved.

\section{Introduction}

Recently, the design and construction of metamaterials with unconventional properties has become an area of active research. Metamaterials are complex composites consisting of an engineered microstructure which is specifically designed to manipulate an applied wave or loading, allowing these materials to display unusual properties such as negative refractive index, negative shear modulus, or negative effective mass. These properties are often desired in applications that deal with electromagnetic,

\footnotetext{
* Corresponding author.

E-mail address: anna.pandolfi@polimi.it (A. Pandolfi).
}

acoustic, or elastic waves, yet are not readily achievable with traditional materials.

Many earlier studies consider the use of metamaterials for the manipulation of electromagnetic waves. There has been significant investigation into the propagation of electromagnetic waves in both one and two-dimensional systems, where analyses include an examination of the photonic band structure of periodic metamaterials utilizing, for example, dielectric scatterers (Sigalas et al., 1993; Smith et al., 1993) and metal cylinders (Smith et al., 1994). Additionally, there has several studies into the use of materials with negative refractive index at optical wavelengths, where examples include the superlenses proposed by Pendry (2000) and the invisibility properties 
demonstrated by Kundtz and Smith (2010) and Urzhumov et al. (2011). This has influenced the development of acoustic metamaterials, and there has been considerable attention placed on the design of sonic or phononic crystals (Kushwaha et al., 1993; Klironomos and Economou, 1998; Psarobas et al., 2000; Goffaux and Sánchez-Dehesa, 2003; Jensen, 2003; Hirsekorn, 2004; Wang et al., 2004a, b,c). Simple phononic crystals involving periodic arrays of cylinders have been shown to exhibit wave filtering behavior. Mártinez-Sala et al. (1995) introduced an investigation into the sound attenuation achieved by a sculpture in Madrid, and this was more formally presented by Sánchez-Pérez et al. (1998) with an experimental analysis of a two-dimensional periodic array of rigid stainless steel cylinders. When these periodic composite materials interact with acoustic waves, they exhibit wave filtering behavior, creating band gaps or stop bands within the frequency spectrum. Within the band gap frequency range, the applied acoustic waves cannot propagate and the incident energy is dispersed or scattered from the medium. These materials often utilize the periodicity of the internal structure to create Bragg-type band gaps (Goffaux and Sánchez-Dehesa, 2003; Hirsekorn, 2004; Wang et al., 2004c). However, certain size restrictions on the dimensions of the acoustic barrier are required to make use of Bragg gaps. To overcome this constraint, Liu et al. (2000) developed an array of locally resonant inclusions, consisting of silicone coated lead spheres in an epoxy matrix. As the sonic wave passes through the material, resonance of the heavy lead core can be activated at chosen frequencies resulting in resonance-induced band gaps.

The wave attenuation behavior of materials with these resonant inclusions is derived from the unusual material properties that these composites display, such as negative effective mass. Mass density is typically considered to be the volume average of the mass of the constituents. However, in the case of resonant inclusions under dynamic excitation, there is relative motion between the constituents and the matrix, which causes the dynamic effective mass density to be different from that found in the static case. Milton and Willis (2007) derive a simple relation for the effective dynamic mass density by considering a rigid bar with hidden voids, each containing a springmass system. The effective mass density for this system is found to be a function of the oscillation frequency and when resonance of the heavy internal mass is activated, the effective mass can become very large and negative in value. This 'negative effective mass' induced by resonance enables systems of this structure to manipulate and reduce the effect of wave energy.

Metaconcrete, recently introduced in Mitchell et al. (2014), represents an example of such a system. Metaconcrete is a modified concrete developed for the purpose of reducing the damage and propagation of energy caused by dynamic loading, such as the blast loading profile generated by an explosion. In a similar way to the extension of hollow cylinder phononic crystals to earthquake mitigation by Brûlé et al. (2014), metaconcrete utilizes the concept of resonance induced negative effective mass that has been successfully used in locally resonant sonic crystals. Metaconcrete employs bi-material spherical inclusions as a replacement for the standard stone and gravel aggregates of regular concrete. These new aggregates modify the response of the system at or near the resonant frequency of the aggregate. Each aggregate contains a heavy core coated in a thin compliant outer layer a few millimeters in thickness. By adjusting the geometry of the aggregate and the coating stiffness we can modify the resonant behavior of the aggregate so that it occurs within a desired frequency range.

Finite element studies of shock loading in metaconcrete slabs consisting of purely elastic constituents have shown that there is a beneficial transfer of energy between the mortar phase and the inclusions when a section of slab is subjected to blast loading, as described in Mitchell et al. (2014). These analyses showed that a significant portion of the total mechanical energy is absorbed by the lead phase of the aggregates in the form of kinetic energy, whereas a much smaller fraction is carried by the mortar component of the metaconcrete slab. This behavior was seen particularly for the aggregate configurations with higher coating elastic moduli, where resonance of the aggregates could be activated under the considered shock loading. For example, in the case of nylon coated lead spheres, approximately $60 \%$ of the total mechanical energy was carried by lead cores while only $30 \%$ was attributed to the mortar. These characteristics of metaconcrete were also seen in the computation of maximum longitudinal stress within the slab, where a significant reduction in mortar stress was seen in the samples with high coating stiffness.

The beneficial transfer of energy and the implication of resonance induced negative effective mass suggest the need to investigate the behavior of a metaconcrete slab at fixed frequencies of applied loading. Transmission ratio plots have been used to quantify the band gap and resonance induced behavior of phononic crystals with an analogously layered structure (Liu et al., 2000; Sheng et al., 2003). These crystals have been shown to exhibit resonant behavior when experimentally tested with sound waves of varying frequencies, where the ratio of the change in wave amplitude across the crystal is measured and plotted against input wave frequency. A similar ratio can be computed for metaconcrete by considering the amount of energy transmitted through a slab and we investigate the effect of resonance by considering this ratio at different forcing frequencies. To this end, we examine the transmission behavior and resonance activation of metaconcrete by considering a slab containing a periodic array of aggregates subjected to a displacement loading over a range of chosen frequencies. The information gained from these analyses allows us to more accurately determine the appropriate aggregate properties for specific loading applications.

The paper is structured as follows. In Section 2 we describe the geometry and material properties used for the modeled aggregates, and two approaches, analytical and numerical, for the computation of aggregate resonant frequencies. The method for computing the transmission ratio from a metaconcrete slab finite element model is presented in Section 3, along with results from the analysis of four aggregate configurations. Concluding remarks are given in Section 4, where we outline other work being 
pursued, such as the integration of brittle behaviors, along with future experimental testing of metaconcrete prototypes.

\section{Aggregate resonant frequency}

Theoretical analyses indicate that metamaterials containing resonant inclusions can trap and prevent the transmission of a propagating wave, particularly at or near the natural frequency of the inclusion. Therefore, we must derive the resonant frequency for metaconcrete by defining the geometry and material properties of the aggregates. The resonant frequency of the aggregate provides an indication of the target frequency range for investigation. The same aggregate configuration is then modeled in periodic arrangement within a metaconcrete slab using finite element analyses, which enables the extraction of energy histories and the computation of transmission coefficients.

We consider the same metaconcrete slab size and aggregate arrangement as investigated previously in Mitchell et al. (2014), with a slab containing a periodic array of aggregates within a homogeneous concrete matrix, as shown in Fig. 1. Metaconcrete aggregates contain two components which interact to cause resonance. Each aggregate within the slab has a layered structure with a spherical lead core coated in a more compliant outer material. For the current study we consider two different geometry configurations, i.e., lead core radii of $R_{l}=11 \mathrm{~mm}$ and $R_{l}=9 \mathrm{~mm}$. These were the largest and smallest lead core sizes considered in the previous study (Mitchell et al., 2014). We also examine two different coating materials, i. e., nylon and rubber, obtaining the four aggregate configurations listed in Table 1.

\subsection{Analytical resonant frequency}

As outlined in Mitchell et al. (2014), we can use a simple one-dimensional spring-mass model to derive a relationship that approximates the resonant frequency of a single aggregate. This expression combines the main geometry and stiffness parameters of the aggregate, accounting for the movement of the central core through the softer outer material. The natural frequency, $f$, of the system, in units of
Table 1

Geometry and material of the aggregates.

\begin{tabular}{lcll}
\hline Configuration & $R_{l}(\mathrm{~mm})$ & $t(\mathrm{~mm})$ & Coating \\
\hline 1 & 11 & 1 & Nylon \\
2 & 9 & 3 & Nylon \\
3 & 11 & 1 & Rubber \\
4 & 9 & 3 & Rubber \\
\hline
\end{tabular}

Hertz, is computed using the properties of the aggregate and is given by

$f=\frac{1}{2 \pi} \sqrt{\frac{3}{2} \frac{E_{c}}{R_{l} t \rho_{l}},}$

where $\rho_{l}$ is the density of the core material, $R_{l}$ is the radius of the heavy core, $t$ is the thickness of the coating, and $E_{c}$ is the Young's modulus of the coating material. This equation gives an approximate value for resonant frequency and thus an indication of the frequency around which investigation of the transmission behavior should occur.

\subsection{Modal analysis of a metaconcrete unit cell}

The aggregate resonant frequency given by Eq. (1) provides an estimate for the purposes of aggregate tuning and design. However, the one-dimensional spring-mass model used to derive this equation cannot adequately capture all the behavior present in the three-dimensional metaconcrete model shown in Fig. 1. To more accurately understand the resonant behavior of the system, we can model a three-dimensional metaconcrete aggregate using commercial finite element software capable of performing frequency or modal analyses (Abaqus/Standard 6.12-2, 2012). We consider a $0.03 \mathrm{~m}$ mortar cube containing a single aggregate, representing a periodic unit cell from a metaconcrete slab. Fig. 2 depicts the finite element model used to estimate the modal properties of a $1 \mathrm{~mm}$ nylon coated aggregate. The model is meshed with quadratic tetrahedral elements with a maximum size of $2.5 \mathrm{~mm}$, reducing to a smaller size within the coating and core phases. Table 2 provides the discretization data for the cases considered, along with the volume fractions for each phase of the aggregate, given as a percentage of total slab volume.

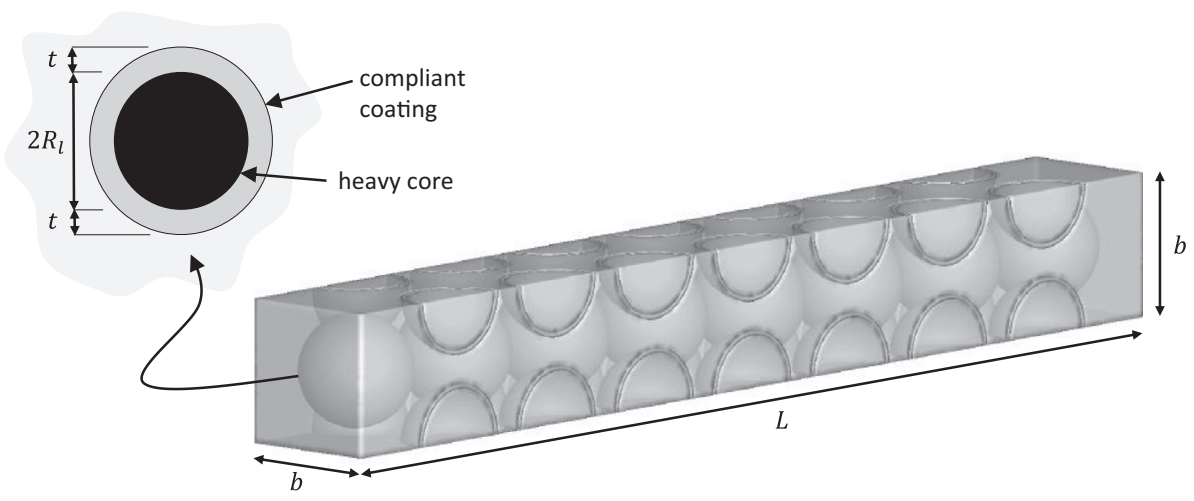

Fig. 1. Solid model for the metaconcrete slab showing the constituents and structure of a metaconcrete aggregate; $R_{l}$ lead core radius, $t$ coating thickness. 


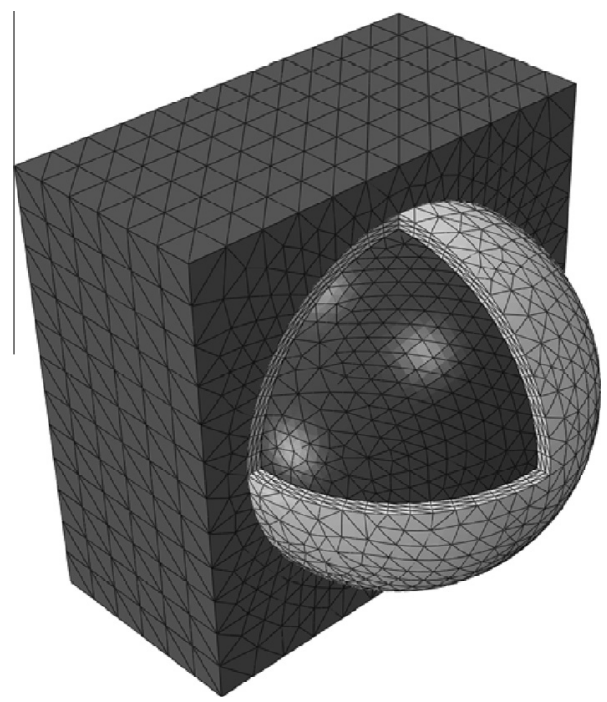

Fig. 2. A periodic unit cell used for the modal analysis, showing the outer mortar block containing a $1 \mathrm{~mm}$ nylon coated lead aggregate.

Boundary conditions are assigned to the outer edges of the cube so that only the modes of interest, those involving the aggregate constituents, are output from the analyses. Each part within the model is assigned the appropriate properties as given in Table 3, where $v$ denotes the Poisson's ratio, and a linear elastic material model is assumed for each constituent.

A frequency analysis is run to compute the first four eigenfrequencies and corresponding eigenshapes. Table 4 lists the results for each aggregate configuration. The first mode shape represents rigid body rotation of the lead core inside the compliant coating. The second mode shape consists of rigid body translation of the heavy core, oscillating within the coating material. The third and forth mode shapes correspond to vibration and flexing of the lead core and/or coating materials.

The second mode shape containing rigid body translation of the heavy core is the mode we wish to activate in metaconcrete and is also the mode shape we hoped to capture in Eq. (1). However, as can be seen from Table 4, while the frequency from Eq. (1) reasonably estimates the first mode natural frequency, it underestimates the second mode resonant frequency by approximately $2-4 \mathrm{kHz}$. Therefore, the additional accuracy provided by the eigenfrequencies from the modal analysis allows us to see which mode shapes produce changes in transmission coefficient. This enables us to more directly attribute the improved transmission ratio to the activation of a

Table 2

Discretization data and volume fractions for the finite element periodic unit cell model.

\begin{tabular}{llllcl}
\hline Configuration & Nodes & Elements & $\begin{array}{l}V_{\text {mortar }} \\
(\%)\end{array}$ & $\begin{array}{l}V_{\text {coating }} \\
(\%)\end{array}$ & $\begin{array}{l}V_{\text {lead }} \\
(\%)\end{array}$ \\
\hline 1 and 3 & 40,307 & 25,047 & 73.2 & 6.2 & 20.6 \\
2 and 4 & 41,074 & 26,260 & 73.2 & 15.5 & 11.3 \\
\hline
\end{tabular}

Table 3

Material constants used in the simulations.

\begin{tabular}{lcll}
\hline Material & $\rho\left(\mathrm{kg} / \mathrm{m}^{3}\right)$ & $E(\mathrm{GPa})$ & $v$ \\
\hline Mortar & 2500 & 30 & 0.20 \\
Lead & 11,400 & 16 & 0.44 \\
Nylon & 1150 & 1.0 & 0.40 \\
Rubber & 900 & 0.01 & 0.49 \\
\hline
\end{tabular}

Table 4

Resonant frequencies from both Eq. (1) and the finite element periodic unit cell model.

\begin{tabular}{lrrrrr}
\hline \multirow{2}{*}{ Configuration } & Eq. (1) $(\mathrm{kHz})$ & \multicolumn{4}{l}{ Finite element unit cell $(\mathrm{kHz})$} \\
\cline { 3 - 6 } & & Mode 1 & Mode 2 & Mode 3 & Mode 4 \\
\hline 1 & 17.41 & 18.84 & 21.12 & 32.14 & 36.42 \\
2 & 11.11 & 15.08 & 15.94 & 34.19 & 36.92 \\
3 & 1.74 & 1.99 & 5.99 & 25.00 & 27.70 \\
4 & 1.11 & 1.51 & 3.39 & 10.44 & 11.12 \\
\hline
\end{tabular}

particular mode of resonance, as will be discussed in the following section.

\section{Computation of wave transmission coefficients from metaconcrete slab models}

Transmission coefficients have been used to identify the band gap properties of both photonic (Sigalas et al., 1993; Smith et al., 1993, 1994) and acoustic crystals (SánchezPérez et al., 1998; Liu et al., 2000; Sheng et al., 2003). For sonic metamaterials using layered resonant inclusions, the change in sound wave amplitude across the crystal was measured experimentally to give the transmission ratio for a chosen wave frequency (Liu et al., 2000; Sheng et al., 2003). A similar ratio can be computed for metaconcrete by considering the amount of mechanical energy transmitted through a slab. This ratio is plotted against input frequency to investigate which ranges of frequency correspond to reduced wave transmission and to ascertain whether this effect is caused by the activation of resonance.

\subsection{Finite element model}

To investigate the transmission of wave energy we employ the same finite element model as used in the elastic material studies, where a metaconcrete slab was subjected to a blast profile generated by an explosion (Mitchell et al., 2014). We consider an infinite planar slab of thickness $L$ containing a periodic array of metaconcrete aggregates and focus on a square cross section of material of dimension $b$ that spans the entire slab thickness. The solid model representation of the metaconcrete slab under analysis is shown in Fig. 1.

The solid model is discretized with a uniform mesh size of $h=1.5 \mathrm{~mm}$, reducing to a smaller value within the coating layer, if necessary. An example of the finite element mesh for an array of $1 \mathrm{~mm}$ coated aggregates is shown in Fig. 3. We can check the maximum allowable applied wave frequency that can by calculated using this mesh by 


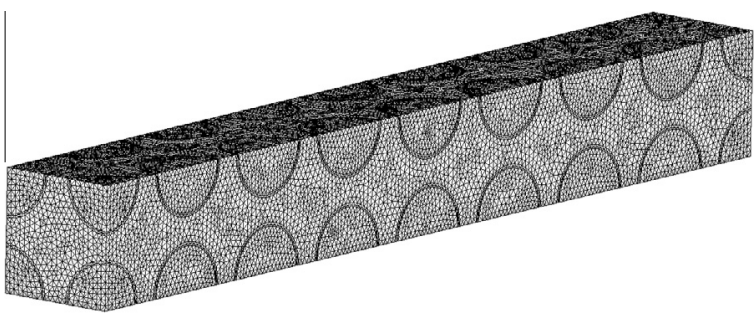

Fig. 3. Finite element discretization (mesh size $h=1.5 \mathrm{~mm}$ ) of a metaconcrete slab containing an array of aggregates with a $1 \mathrm{~mm}$ coating.

considering the maximum wavenumber $k_{\max }$ computed using the mesh size $h$,

$k_{\max }=\frac{1}{h}$

We obtain the equation for maximum frequency $f_{\max }$ by multiplying the maximum wavenumber by the longitudinal wave speed of the material $c_{L}$,

$f_{\max }=\frac{c_{L}}{h}$.

If we consider the longitudinal wave speed for the homogeneous mortar material, $c_{L}=3464 \mathrm{~m} / \mathrm{s}$, given by $c_{L}=\sqrt{E / \rho}$, we compute the approximate maximum allowable frequency as $f_{\max }=2309 \mathrm{kHz}$.

The chosen dimensions for the slab are $L=0.24 \mathrm{~m}$ and $b=0.03 \mathrm{~m}$, with a periodic arrangement of aggregates at $0.03 \mathrm{~m}$ centers. This gives a total of 8 whole spherical central inclusions and 28 quarter spheres around the longitudinal edges of the slab. Each aggregate has an outer diameter of $24 \mathrm{~mm}$ and contains a central lead core.

The four aggregate configurations presented in Table 1 are used to investigate the effect of different material and geometry selections on wave transmission and resonance activation within the slab. In addition, we consider two periodic aggregate arrays. First, the slab is configured with a total of 8 aggregates in a periodic arrangement along the center of the slab. The simulations are then run again with a more densely packed configuration, this time also including the 28 quarter spheres surrounding the outer edges of the slab, giving a total of 36 separate inclusions. A mortar slab with no inclusions is analyzed as a baseline comparison. The discretization data for the finite element models is given in Table 5, along with the volume fractions for both aggregate arrays presented for each constituent as a percentage of total slab volume.

The material constants used in the simulations are collected in Table 3. We consider purely elastic behavior for all components, with the material behavior described by a neo-Hookean material model, extended to the compressible range. The forcing function is generated by applying a sinusoidal displacement to all nodes on one end face of the slab at a prescribed frequency. The other end face is unconstrained, and we apply periodic boundary conditions to the lateral faces of the slab. To have the same input energy for every harmonic wave frequency, we scale the amplitude of the applied displacement relative to a reference resonance value. The energy of a harmonic wave is proportional to the square of both the frequency and the amplitude of the wave, i.e.,

$E \propto \omega^{2} A^{2}$

To maintain the same input energy for a frequency other than the resonant value, we therefore multiply the reference amplitude by $f_{r} / f$, the ratio of the resonant frequency relative to the frequency value under consideration. For example, an amplitude of $A_{r}=0.001 \mathrm{~m}$ was chosen for the reference critical frequency equal to the resonant frequency of the inclusion. The amplitude for a subcritical frequency $f_{1}=0.25 f_{r}$ is therefore $A_{1}=0.004 \mathrm{~m}$, and similarly, the amplitude for a supercritical frequency $f_{2}=2 f_{r}$ is $A_{2}=0.0005 \mathrm{~m}$.

The amount of energy trapped within the metaconcrete aggregates is averaged over a total fixed time duration for each configuration. We begin computation of the transmission ratio after the greater of either one period of the harmonic forcing function or the time it takes for approximately two transits of a wave through a homogeneous slab. The transit time for the wave to travel once through the length of the slab is given by $t_{L}=L / c_{L}$, where $L$ is the length of the slab and $c_{L}=3464 \mathrm{~m} / \mathrm{s}$ is the longitudinal wave speed of mortar, given previously. For the homogeneous mortar slab under consideration this gives $t_{L}=7 \times 10^{-5} \mathrm{~s}$ and a minimum time $2 t_{L}=1.4 \times 10^{-4} \mathrm{~s}$. The end time for the simulation is chosen based on the aggregate configuration and is given by $t_{\text {total }}=0.0004 \mathrm{~s}$ for the $1 \mathrm{~mm}$ nylon coated aggregates, $t_{\text {total }}=0.0005 \mathrm{~s}$ for the $3 \mathrm{~mm}$ nylon coated aggregates, $t_{\text {total }}=0.0011 \mathrm{~s}$ for the $1 \mathrm{~mm}$ rubber coated aggregates, and $t_{\text {total }}=0.0018 \mathrm{~s}$ for the $3 \mathrm{~mm}$ rubber coated aggregates. The time interval for averaging is chosen so that there is a minimum of two transits of the applied wave through the slab, with an average of 15 cycles of the applied displacement over the duration of the calculation. This allows sufficient time for resonance activation of the aggregates while preventing any build-up of numerical interference.

To compute the transmission coefficient we consider the amount of energy that is trapped by the aggregate farthest away from the applied loading and compare this to the behavior of the entire slab. This indicates how much energy reaches the far end of the slab and therefore how much energy has be distributed and absorbed by the aggregates during the transition of the wave. The transmission coefficient $T$ is therefore given by the ratio of the amount of energy trapped in the last aggregate relative to the total energy trapped within all the aggregates of the slab,

$T=\frac{E^{N}}{\sum_{j=1}^{N} E^{j}}$,

where $E^{j}$ is the total mechanical energy of the $j$ th aggregate, time-averaged over the duration of the calculation, and $N$ is the total number of aggregates, where the aggregate farthest from the exposed face is denoted by $j=N$. A decrease in transmission coefficient therefore implies that 
Table 5

Discretization data and constituent volume fractions

\begin{tabular}{|c|c|c|c|c|c|c|}
\hline Configuration & Aggregates & Nodes & Elements & $V_{\text {mortar }}(\%)$ & $V_{\text {coating }}(\%)$ & $V_{\text {lead }}(\%)$ \\
\hline 1 and 3 & 8 & 102,965 & 561,846 & 73.2 & 6.2 & 20.6 \\
\hline 2 and 4 & 8 & 84,000 & 451,457 & 73.2 & 15.5 & 11.3 \\
\hline 1 and 3 & 36 & 102,965 & 561,846 & 49.7 & 11.6 & 38.7 \\
\hline 2 and 4 & 36 & 84,000 & 451,457 & 49.7 & 29.1 & 21.2 \\
\hline
\end{tabular}

less energy has reached the final aggregate and thus activation of the inclusions has resulted in a behavior that absorbs energy from the applied wave.

\subsection{Transmission plots for four aggregate configurations}

The transmission ratio is computed from the results of the finite element analyses using the model and material properties described in the previous section. Both the 8 and 36 aggregate array configurations are tested for each model with the same range of applied wave frequencies, varying between 4 and $139 \mathrm{kHz}$ for the $1 \mathrm{~mm}$ nylon coated inclusions, $5-100 \mathrm{kHz}$ for the $3 \mathrm{~mm}$ nylon coated inclusions, $2-37 \mathrm{kHz}$ for the $1 \mathrm{~mm}$ rubber coated inclusions, and $1-22 \mathrm{kHz}$ for the $3 \mathrm{~mm}$ rubber coated inclusions. Each frequency range is chosen so that any behavior near the four modes of vibration given in Table 4 can be fully captured. In addition, the maximum frequency considered is selected to be significantly smaller than the maximum allowable frequency computed from Eq. (3).

The resulting plots for the two cases of nylon coated lead inclusions are shown in Fig. 4. The plot for the $1 \mathrm{~mm}$ nylon coated inclusions, shown in Fig. 4(a), displays a low frequency dip in transmission coefficient at $22 \mathrm{kHz}$ for both the 8 and 36 aggregate arrays. This dip occurs near the approximate resonant frequency of $17.4 \mathrm{kHz}$ calculated from Eq. (1) and at the Mode 2 resonant frequency of $21.12 \mathrm{kHz}$ computed with the modal analysis.

The transmission plot also displays a second dip at a higher frequency of $35 \mathrm{kHz}$, which corresponds approximately to the Mode 3 and 4 frequencies found from the modal analysis. This dip is slightly greater in magnitude and wider in frequency range, which indicates that vibration and oscillation of the core and coating at higher frequencies produces a greater reduction in transmission. However, a distinct dip is only apparent for the 36 aggregate case and a significant drop in transmission ratio is not seen when fewer aggregates are present in the slab. A further decrease at very high frequencies greater than approximately $78 \mathrm{kHz}$ is also observed. The highfrequency decrease in transmission coefficient seen in Fig. 4(a) is possibly due to the periodic arrangement of aggregates combining to provide amplification of the energy absorption properties, a Bragg-type scattering of the waves caused by the applied wavelength nearing the aggregate spacing and dimensions, improved performance at higher modal frequencies, or numerical interference.

We observe an improvement in transmission ratio when we compare the results of the metaconcrete configurations to that achieved with a homogeneous mortar slab. The transmission coefficient for the homogeneous slab is independent of the frequency of applied loading,

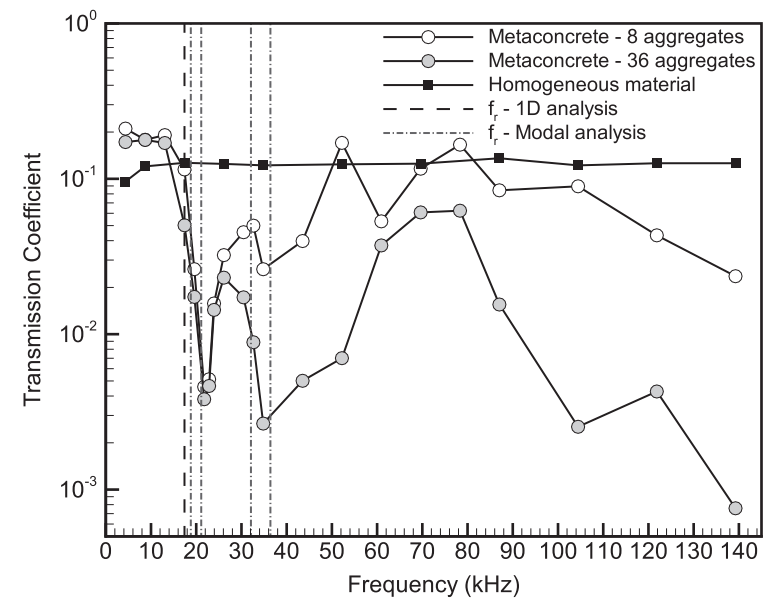

(a)

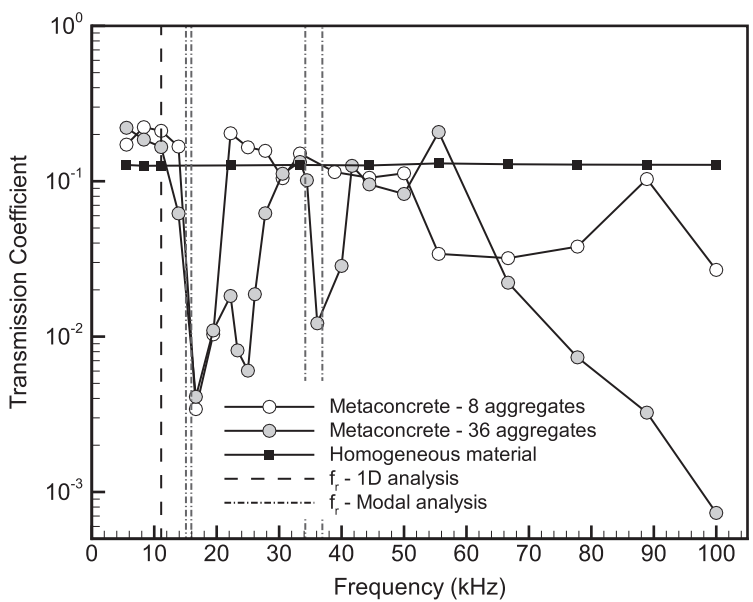

(b)

Fig. 4. Transmission coefficient plotted against frequency of excitation for a metaconcrete slab consisting of (a) $1 \mathrm{~mm}$ and (b) $3 \mathrm{~mm}$ nylon coated aggregates. Also shown are the corresponding transmission coefficients for a homogeneous slab along with the resonant frequencies, $f_{r}$, of the inclusion from both the approximate equation and the modal analysis.

maintaining a value of 0.13 over the entire frequency range for all cases. The low frequency dip for the $1 \mathrm{~mm}$ nylon aggregate at $22 \mathrm{kHz}$ displays a magnitude that is $3.0 \%$ of the transmission coefficient of the homogeneous mortar slab for both aggregate configurations. The second low frequency dip for the 36 aggregate arrangement occurs at $35 \mathrm{kHz}$, with $2.2 \%$ of the mortar value. This improvement over the homogeneous mortar slab suggests enhanced performance within the range of frequencies corresponding to the dips. 
We see similar behavior for the $3 \mathrm{~mm}$ nylon aggregate case, shown in Fig. 4(b). A sharp dip for both array configurations is located at the Mode 2 natural frequency of the aggregate, $15.94 \mathrm{kHz}$. The magnitude of this dip is $3.2 \%$ of the homogeneous mortar slab coefficient, thus achieving a similar Mode 2 dip magnitude to the $1 \mathrm{~mm}$ nylon case. Like the $1 \mathrm{~mm}$ nylon aggregate case, we also observe a higher frequency dip for the 36 aggregate configuration located near the Mode 3 and 4 natural frequencies, along with a drop in transmission coefficient at very high frequencies greater than $60 \mathrm{kHz}$.

The results for the two rubber coated aggregate configurations are shown in Fig. 5. The $1 \mathrm{~mm}$ rubber coated aggregate case, shown in Fig. 5(a), displays a dip located in the range of $6-10 \mathrm{kHz}$, which corresponds to the Mode 2 natural frequency of $5.99 \mathrm{kHz}$ as computed by the modal analysis. This dip occurs for both the 8 and 36 aggregate

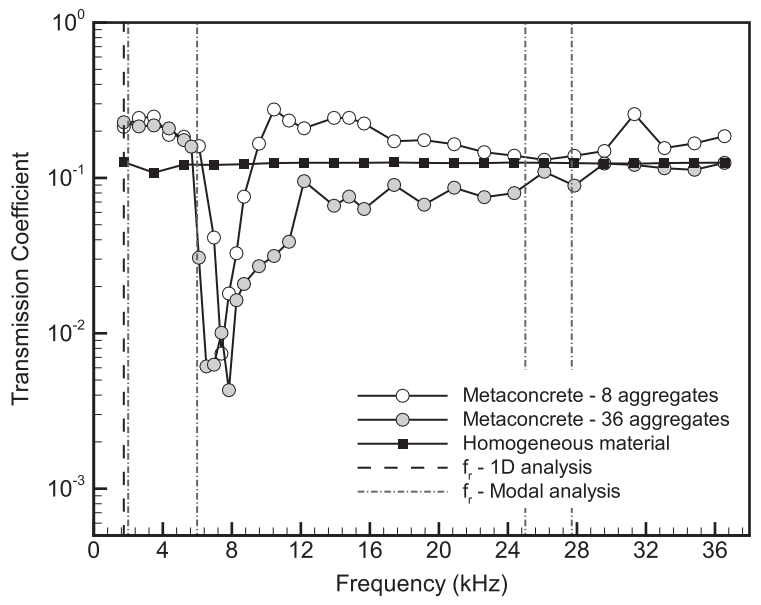

(a)

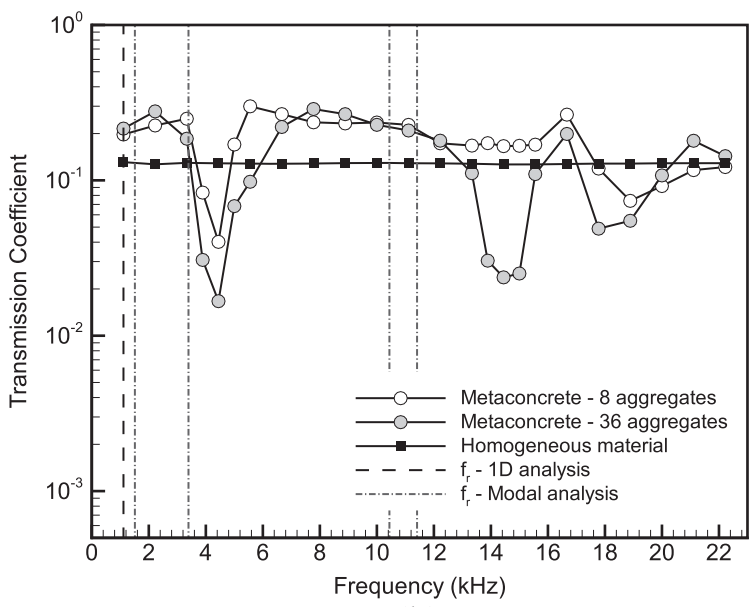

(b)

Fig. 5. Transmission coefficient plotted against frequency of excitation for a metaconcrete slab consisting of (a) $1 \mathrm{~mm}$ and (b) $3 \mathrm{~mm}$ rubber coated aggregates. Also shown are the corresponding transmission coefficients for a homogeneous slab along with the resonant frequencies, $f_{r}$, of the inclusion from both the approximate equation and the modal analysis. configurations and reaches $3.5 \%$ of the homogeneous mortar value, although the apparent band gap is wider when there are more aggregates present in the slab. For either array configuration case, we do not observe a second dip at higher frequencies near the Mode 3 and 4 values. Instead, the transmission ratio sits near that of the homogeneous mortar slab, indicating a lack of improved performance at higher frequencies for the case where a thin $1 \mathrm{~mm}$ rubber coating is used.

By contrast, the transmission plot for the $3 \mathrm{~mm}$ rubber coated aggregates, shown in Fig. 5(b), displays three distinct dips in transmission coefficient, most particularly for the 36 aggregate arrangement. The first dip occurs in the frequency range of $3.5-5.5 \mathrm{kHz}$ and is present for both the 8 and 36 aggregate cases, achieving a minimum of $13.0 \%$ of the homogeneous slab transmission value. The second dip is present only in the 36 aggregate configuration and occurs in the range of $14-16 \mathrm{kHz}$, reaching $18.8 \%$ of the homogeneous mortar value. The third dip is present for both aggregate array configurations and occurs in the range of $17-21 \mathrm{kHz}$. Only the first dip corresponds directly to the natural frequency computed with the modal analysis, occuring near the Mode 2 natural frequency. The second and third dips are greater than $3 \mathrm{kHz}$ higher than the fourth Mode natural frequency, suggesting they are generated by some higher mode combination of coating and core oscillation within the aggregate.

The three gaps observed in the transmission plot in Fig. 5(b) for the $3 \mathrm{~mm}$ rubber case are more distinct than any other configuration of coating material and thickness. However, the magnitude of the dips are much smaller and the gaps are narrower, or in the case of the 8 aggregate configuration, barely present. Furthermore, the shape of the gaps present in Fig. 5(b) also resemble the form of the measured amplitude transmission found in the experiments conducted by Liu et al. (2000) and Sheng et al. (2003) on sound waves in locally resonant sonic crystals. Sound wave transmission was tested using an array of $15 \mathrm{~mm}$ diameter inclusions with $2.5 \mathrm{~mm}$ silicone rubber coated lead cores. These inclusions are of similar configuration to those tested in the metaconcrete slab and the transmission coefficient plots produced from experiments on acoustic crystals of this structure display a similar trend; two distinct dips in transmission coefficient with frequency locations corresponding to those calculated from theoretical analyses.

For the rubber coated aggregates we note that there are large ranges in frequency for which the plots indicate that metaconcrete performs the same or less favorably than the homogeneous concrete slab; the transmission coefficient sits above the line of the reference homogeneous value. In contrast, the aggregates with the comparatively more stiff nylon coating, in general, perform better than the concrete slab over large spans of frequency within the range under investigation.

We also note that a reduction in the transmission ratio implies a concentration of energy within the aggregates near the front of the slab, which leads to a corresponding increase in stress within the activated aggregates. The distribution of longitudinal Cauchy stress within a metaconcrete slab was explored in Mitchell et al. (2014) and the 
transmission calculations correlate with the results observed in these analyses. In Mitchell et al. (2014) it was shown that metaconcrete aggregates can absorb a large fraction of the supplied energy and this effect was reflected in the slab stress distribution, where the activation of aggregates led to a significantly reduced amount of stress propagating through the slab and a more widely spread stress wave. However, in the metaconcrete slab the stress was also concentrated in the area near the face exposed to the applied loading. The transfer of energy observed in both the transmission and shock mitigation analyses therefore indicates that the corresponding transfer of stress between the mortar and the inclusions may lead to weakening and damage in the mortar surrounding the front aggregates. This effect is explored further in Mitchell et al. (in press), where we conduct analyses on a metaconcrete slab under erosion by incorporating the fracture behavior of the mortar matrix.

The transmission coefficient plots indicate the existence of bands of reduced energy transmission within the frequency spectrum and also suggest a frequency range for which a particular aggregate configuration provides the most beneficial behavior. For each of the four cases, the most significant dip in transmission coefficient is achieved near the Mode 2 natural frequency computed by the modal analysis. This dip is present for both 8 and 36 aggregate configurations, with both aggregate arrays achieving the same dip magnitude in the Mode 2 range in three out of four cases. For the nylon coated inclusion, this gap is given by frequencies within the range of approximately 20$60 \mathrm{kHz}$ for the $1 \mathrm{~mm}$ coated aggregates and $13-30 \mathrm{kHz}$ for the $3 \mathrm{~mm}$ coated aggregates. For the more compliant rubber coating, the Mode 2 gap is present in the range of $6-9 \mathrm{kHz}$ for the $1 \mathrm{~mm}$ coated aggregates and $3.5-5.5 \mathrm{kHz}$ for the $3 \mathrm{~mm}$ coated aggregates. The understanding gained from the location of these frequency bands for each inclusion configuration can be further utilized in the design of metaconcrete aggregates for loading applications where attenuation of dynamic excitation with a specific frequency profile is desired.

\section{Conclusions}

We present a numerical investigation of the wave energy transmission behavior of four metaconcrete slabs for a range of forcing frequencies. Metaconcrete is a new type of engineered metamaterial for dynamic loading applications, where the standard stone and gravel aggregates of regular concrete are replaced by designed inclusions that resonate at chosen frequencies. The activation of resonance leads to what as known as 'negative effective mass', which has been demonstrated by the theoretical studies of Milton and Willis (2007). Phononic metamaterials with resonant inclusions have also used this behavior to manipulate the propagation of sound waves, and experimental tests on sonic crystals have produced transmission plots that demonstrate dips near resonant frequencies, indicating the location of frequency bands where strong sound attenuation is achieved.
Investigation into the behavior of metaconcrete slabs with elastic constituents in Mitchell et al. (2014) suggested the need to consider the frequency-dependent nature of metaconcrete in order to further understand aggregate design. Therefore, a finite element model is used to compute the transmission coefficients for metaconcrete slabs over a range of forcing frequencies. The transmission coefficients are computed based on the amount of energy transmitted through the slab, where the average energy absorbed by the last aggregate was compared to that of all the aggregates. The model is analyzed with array arrangements of either 8 or 36 coated aggregates and the study considers four different aggregate configurations; $1 \mathrm{~mm}$ nylon, $3 \mathrm{~mm}$ nylon, $1 \mathrm{~mm}$ rubber, and $3 \mathrm{~mm}$ rubber coated inclusions.

The results reveal dips in the transmission coefficient near the rigid body translation resonant frequency of the inclusion. We see a second dip in three out of four cases, located at or near the higher mode vibration and flexing of the core and coating material. Within the dip frequency range, the magnitude of energy transmission is greatly reduced when compared to that observed in a homogeneous mortar slab. This improvement in performance over a slab with no resonant aggregates suggests ranges of frequency that will provide the most beneficial behavior for each aggregate configuration. The density of the aggregates within the slab is also seen to affect the magnitude of the dip in transmission coefficient. A larger number of aggregates per unit volume allows for a greater reduction in transmission, and more significantly, a wider frequency range of influence. Of the two cases tested, aggregates with the more stiff nylon coating provide a greater dip in wave energy transmission over a larger range of applied frequencies. As in the blast wave loading application considered in Mitchell et al. (2014), the study of energy transmission suggests that a nylon coating is the best choice for an overall improvement in the behavior of metaconcrete with elastic constituents.

The present study, along with previous work, indicates that metaconcrete is capable of improving performance under dynamic loading when compared with conventional concrete. However, the models considered thus far only utilize periodic arrays of metaconcrete aggregates. In the practical implementation of metaconcrete as a structural material it is possible that the aggregates could be mixed into mortar in the same way as the stone and gravel aggregates of regular concrete. In this case a periodic array of aggregates would not easily be achieved. The use of random aggregate arrangements may alter the transmission behavior of metaconcrete, possibly changing the magnitude and width of the dips in transmission coefficient. Further investigation of non-periodic aggregate arrays would therefore provide greater depth of insight into the behavior of metaconcrete slabs and the performance of metaconcrete when used for structural purposes.

The transmission analyses conducted also only consider elastic constituents and do not include the effect of brittle fracture. Concurrent numerical calculations incorporating the fracture characteristics of the mortar matrix confirm similar wave propagation behavior to the elastic analyses 
(Mitchell et al., in press). The results reveal erosion of the slab surface closest to the applied loading, causing the stress to be concentrated around the exposed face and within the aggregates near the front of the slab, resulting in shielding of the slab interior from the shock wave.

Experimental validation of the numerical results would also provide further insight into the resonant behavior of the aggregates and the effect this has on the performance of the overall material. An experiment could be designed to test the resonant properties of a single aggregate, comparing the resonant frequency to that found in an equivalent numerical investigation. Further, a full scale sample of a metaconcrete slab could be fabricated with an array of internal aggregates. The transmission of wave amplitude or energy through a metaconcrete specimen could be measured by testing the specimen with an applied loading over a range of known forcing frequencies. This experimental evaluation of transmission behavior would allow for comparison with the attenuation observed in the numerical calculations so far investigated.

These areas suggest worthwhile avenues for future investigation of metaconcrete behavior and, along with the transmission studies conducted here, will provide greater understanding of the design and potential uses for metaconcrete as a alternative construction material for blast wave, seismic, and impact applications.

\section{Acknowledgments}

This research was supported by the Air Force Office of Scientific Research Grant \# FA9550-12-1-0091 through the University Center of Excellence in High-Rate Deformation Physics of Heterogeneous Materials and is gratefully acknowledged.

\section{References}

Abaqus/Standard 6.12-2, 2012. Dassault Systémes Simulia Corp, Providence, RI.

Brûlé, S., Javelaud, E.H., Enoch, S., Guenneau, S., 2014. Experiments on seismic metamaterials: molding surface waves. Phys. Rev. Lett. 112 (13), 133901

Goffaux, C., Sánchez-Dehesa, J., 2003. Two-dimensional phononic crystals studied using a variational method: application to lattices of locally resonant materials. Phys. Rev. B 67 (14), 144301.
Hirsekorn, M., 2004. Small-size sonic crystals with strong attenuation bands in the audible frequency range. Appl. Phys. Lett. 84 (17), $3364-$ 3366.

Jensen, J.S., 2003. Phononic band gaps and vibrations in one- and twodimensional mass-spring structures. J. Sound Vib. 266 (5), 10531078.

Klironomos, A.D., Economou, E.N., 1998. Elastic wave band gaps and single scattering. Solid State Commun. 105 (5), 327-332.

Kundtz, N., Smith, D.R., 2010. Extreme-angle broadband metamaterial lens. Nat. Mater. 9 (2), 129-132.

Kushwaha, M.S., Halevi, P., Dobrzynski, L., Djafari-Rouhani, B., 1993. Acoustic band structure of periodic elastic composites. Phys. Rev. Lett. 71 (13), 2022-2025.

Liu, Z., Zhang, X., Mao, Y., Zhu, Y.Y., Yang, Z., Chan, C.T., Sheng, P., 2000. Locally resonant sonic materials. Science 289 (5485), 1734-1736.

Mártinez-Sala, R., Sancho, J., Sánchez, J.V., Gómez, V., Llinares, J. Meseguer, F., 1995. Sound attenuation by sculpture. Nature 378, 241.

Milton, G.W., Willis, J.R., 2007. On modifications of newton's second law and linear continuum elastodynamics. Proc. Roy. Soc. A 463 (2079), 855-880.

Mitchell, S.J., Pandolfi, A., Ortiz, M., 2014. Metaconcrete: designed aggregates to enhance dynamic performance. J. Mech. Phys. Solids 65, 69-81.

Mitchell, S. J., Pandolfi, A., Ortiz, M., in press. Effect of brittle fracture in a metaconcrete slab under shock loading. J. Eng. Mech., pp. 1-34.

Pendry, J.B., 2000. Negative refraction makes a perfect lens. Phys. Rev. Lett. 85 (18), 3966-3969.

Psarobas, I.E., Stefanou, N., Modinos, A., 2000. Scattering of elastic waves by periodic arrays of spherical bodies. Phys. Rev. B 62 (1), 278-291.

Sánchez-Pérez, J.V., Caballero, D., Mártinez-Sala, R., Rubio, C., SánchezDehesa, J., Meseguer, F., Llinares, J., Gálvez, F., 1998. Sound attenuation by a two-dimensional array of rigid cylinders. Phys. Rev. Lett. 80 (24), 5325-5328.

Sheng, P., Zhang, X.X., Liu, Z., Chan, C.T., 2003. Locally resonant sonic materials. Phys. B: Condens. Matter 338 (1-4), 201-205.

Sigalas, M., Soukoulis, C.M., Economou, E.N., Chan, C.T., Ho, K.M., 1993. Photonic band gaps and defects in two dimensions: studies of the transmission coefficient. Phys. Rev. B 48 (19), 14121-14126.

Smith, D.R., McCall, S.L., Platzman, P.M., Dalichaouch, R., Kroll, N., Schultz, S., 1993. Photonic band structure and defects in one and two dimensions. J. Opt. Soc. Am. B 10 (2), 314-321.

Smith, D.R., Schultz, S., Kroll, N., Sigalas, M., Ho, K.M., Soukoulis, C.M., 1994. Experimental and theoretical results for a two-dimensional metal photonic band-gap cavity. Appl. Phys. Lett. 65 (5), 645-647.

Urzhumov, Y.A., Kundtz, N.B., Smith, D.R., Pendry, J.B., 2011. Cross-section comparisons of cloaks designed by transformation optical and optical conformal mapping approaches. J. Opt. 13 (2), 024002.

Wang, G., Wen, J., Liu, Y., Wen, X., 2004a. Lumped-mass method for the study of band structure in two-dimensional phononic crystals. Phys. Rev. B 69 (18), 184302.

Wang, G., Wen, X., Wen, J., Shao, L., Liu, Y., 2004b. Two-dimensional locally resonant phononic crystals with binary structures. Phys. Rev. Lett. 93 (15), 154302.

Wang, G., Yu, D., Wen, J., Liu, Y., Wen, X., 2004c. One-dimensional phononic crystals with locally resonant structures. Phys. Lett. A 327 (5-6), 512-521. 